\title{
Nonselective Bilateral Embolization of Internal Iliac Arteries with N-Butyl-2-Cyanoacrylate in Hemodynamically Unstable Patients with Pelvic Fracture
}

\author{
1) Department of Radiology, St. Marianna University School of Medicine, Japan \\ 2) Department of Emergency and Critical Care Medicine, St. Marianna University School of Medicine, Japan
}

Takafumi Haraguchi ${ }^{1)}$, Shingo Hamaguchi ${ }^{2)}$

\begin{abstract}
Purpose: This study was designed to evaluate the efficacy and safety of nonselective bilateral embolization of the internal iliac arteries (IIAs) with n-butyl-2-cyanoacrylate (NBCA) in hemodynamically unstable patients with pelvic fractures.

Material and Methods: Twelve patients underwent nonselective bilateral embolization of the IIAs using NBCA diluted with lipiodol at our institution between January 2004 and March 2014. We analyzed the time of bilateral occlusion of the IIAs, the time from admission to entrance into the interventional radiology room, the need for repeat embolization, outcomes, cause of death, follow-up period, and complications.

Results: The mean duration of bilateral occlusion of the IIAs was 17 min (range, 4-34 min), and the mean time from admission to entrance into the interventional radiology room was 89 min (range, 28-168 min). All patients underwent technically successful embolization. Repeat embolization was required after treatment in three patients. The mortality rate was $33.3 \%$. Complications after embolization were suspected in one patient.

Conclusions: Nonselective bilateral embolization of IIAs with NBCA could be a choice of treatment for hemodynamically unstable patients with severe pelvic fracture hemorrhage.
\end{abstract}

Key words: pelvic fracture, trauma, TAE, NBCA

(Interventional Radiology 2021; 6: 37-43)

\section{Introduction}

Pelvic fracture with significant hemorrhage is often the result of high-energy trauma, such as traffic accidents or falls from a height, and is associated with a high mortality rate [1]. In hemodynamically unstable patients with pelvic fracture, the internal iliac artery (IIA) is often the source of bleeding, and transarterial embolization (TAE) is an effective treatment $[2,3]$. Selective embolization is performed when bleeding is localized and when time permits, whereas nonselective embolization is commonly performed in patients with multiple bleeding sites and is less time-consuming [4]. Nonselective bilateral embolization is also performed for patients with severe injury involving multiple bleeding sites and is a widely accepted procedure without serious complications [5].

Patients with multiple injuries require immediate hemostasis and considerable care for trauma-induced coagulopathy, which is associated with dilution, hypothermia, acidemiaassociated dysfunction, or consumption of coagulation proteases, and the injury itself [6]. Velmahos et al. [7] reported that nonselective embolization of IIAs with gelatin sponge was not effective in some patients with coagulopathy and hemorrhage. In contrast, another study showed that n-butyl2-cyanoacrylate (NBCA) diluted with Lipiodol (NBCA-Lip) was more effective and feasible than gelatin sponge particles in TAE for achieving hemostasis in patients with coagulopa- 


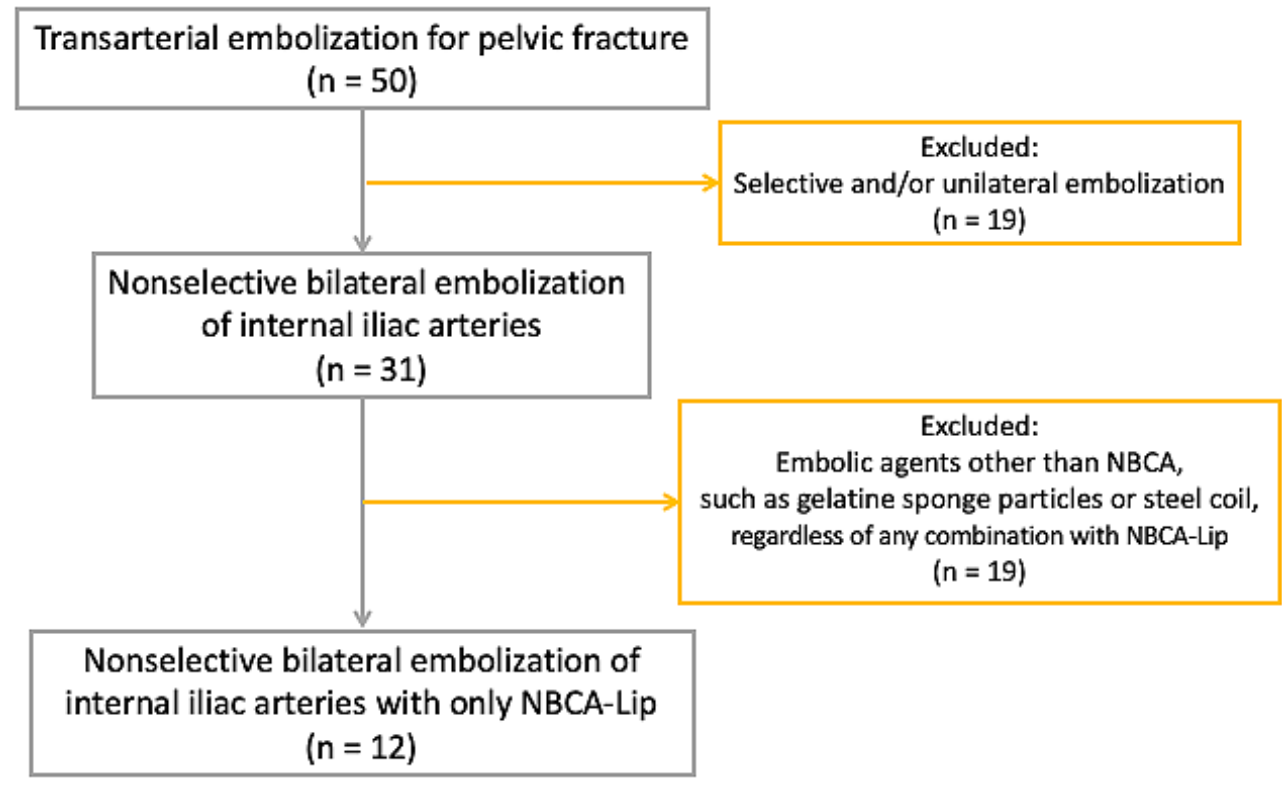

Figure 1. Flow chart of patient selection. Of the $\mathbf{5 0}$ patients who underwent transarterial embolization, we excluded 19 patients in whom selective and/or unilateral embolization was performed and 19 patients treated with embolic agents other than n-butyl-2-cyanoacrylate (NBCA) diluted with Lipiodol (NBCA-Lip), such as gelatin sponge particles or steel coil, regardless of any combination with NBCA-Lip. The remaining 12 patients who underwent nonselective bilateral embolization of the internal iliac arteries with only NBCA-Lip were included.

thy [8-11]. Based on these findings, we have often used NBCA-Lip for active bleeding in patients with coagulopathic conditions in various situations. Particularly, in hemodynamically unstable and coagulopathic patients with pelvic fracture, we usually perform nonselective bilateral embolization with NBCA-Lip.

To our knowledge, no study has been conducted to examine the efficacy and safety of nonselective bilateral embolization of IIAs with NBCA-Lip in patients with pelvic fractures.

\section{Material and Methods}

\section{Patient selection}

This retrospective study was performed to identify and analyze all cases of non-selective embolization of bilateral IIAs with NBCA-Lip performed at our institution from January 2004 to March 2014 to treat hemorrhage resulting from pelvic fracture. Our search revealed 50 consecutive patients who underwent TAE for pelvic fractures caused by blunt trauma. We excluded 19 patients who underwent selective embolization and/or unilateral embolization of the IIA. Another 19 patients were excluded because other embolization agents were used (gelatin sponge particles or steel coil), regardless of any combination with NBCA-Lip. Thus, this retrospective analysis included 12 patients who underwent non-selective bilateral embolization of the IIAs with NBCALip alone (Fig. 1). The study design was approved by our institutional review board. The requirement for informed consent was waived because of the retrospective nature of the study.

\section{General management (of serious traumatic injuries) and indication for TAE}

Patients with serious traumatic injuries and shock underwent a clinical assessment, chest and pelvic plain radiographic examination, and focused assessment with sonography. Patients who responded to initial fluid resuscitation underwent contrast-enhanced computed tomography (CECT) to detect signs of active bleeding and organ injuries and to determine the most effective treatment plan. However, hemodynamically unstable patients who did not respond to resuscitation efforts underwent damage-control surgery or TAE in the emergency department without CECT in select cases.

The indications for TAE for retroperitoneal hemorrhage secondary to pelvic fracture were signs of active bleeding on CECT, such as contrast extravasation and a pseudoaneurysm; signs suspicious for active bleeding, such as dilation of arteries on CECT in hemodynamically unstable patients, or pelvic fracture on a pelvic radiograph in hemodynamically unstable patients who could not be moved to the CT room. Nonselective embolization was performed when angiography showed multiple bleeding arteries or multiple vessel injuries in hemodynamically unstable patients. The indications for NBCA were coagulopathy or bleeding disorders requiring immediate treatment at the operator's discretion.

\section{Data analysis}

The examined data included age, injury, coagulopathy, 
Abbreviated Injury Scale score, Injury Severity Score (ISS), contrast extravasation, arteries with contrast extravasation, organs with concomitant injury, clinical status, and preoperative findings. Coagulopathy was defined as an international normalized ratio $>1.5$, partial thromboplastin time $>45 \mathrm{~s}$, platelet count $<80,000 / \mu \mathrm{L}$, and fibrinogen level $<200 \mathrm{mg} / \mathrm{dL}$ [12].

The evaluation parameters included the time required for bilateral occlusion of the IIAs, time from admission to entry into the interventional radiology (IR) room, NBCA dilution rate, injection dose, repeat embolization, treatment outcome, cause of death, follow-up period, and complications. The time required for bilateral occlusion of the IIAs was defined as the total treatment time from the digital subtraction angiography (DSA) examination before NBCA-Lip injection to the final DSA examination in which hemostasis of the bilateral IIAs was confirmed.

\section{Embolization technique}

All TAE procedures underwent with common femoral arterial approach. A 4- or 5-Fr sheath (Medikit Super Sheath; Medikit Co., Tokyo, Japan) was inserted into the common femoral artery. Pelvic arteriography was initially performed using a 4-Fr pigtail catheter in the terminal aorta. The pigtail catheter was then removed, and a 4- or 5-Fr diagnostic catheter was inserted into the contralateral IIA as the most expedient method. Immediately after pelvic arteriography or internal iliac arteriography, the bleeding sites and types of arterial injuries were evaluated. When multiple distal bleeding sites were identified, non-selective IIA embolization with gelatin sponge particles was preferred to save time. Furthermore, in patients with abnormal coagulation function or requiring immediate treatment, non-selective IIA embolization with NBCA-Lip was performed. In these cases, a microcatheter ( $\mu 7$; Terumo Corporation, Tokyo, Japan) was then advanced into the IIA using a coaxial technique. The microcatheter tip was positioned above the bifurcation of the superior gluteal artery to avoid overflow into the external iliac artery. Immediately before injection of NBCA-Lip, the microcatheter was flushed with approximately $2.0 \mathrm{~mL}$ of $5 \%$ dextrose solution to prevent early polymerization of NBCA. Next, a solution of $1 / 4$ to $1 / 8$ NBCA-Lip was injected using DSA. The low NBCA-Lip ratio (up to 1/4) was used for the purpose of spreading diffusely depending on the operator's decision. When the NBCA-Lip had spread adequately, the microcatheter was retracted to prevent catheter attachment to the arterial wall and was subsequently flushed with a solution of Lipiodol and/or 5\% dextrose to prevent microcatheter obstruction with the NBCA-Lip. The diagnostic catheter was then inserted into the ipsilateral IIA, and the microcatheter was placed in the same position as the contralateral side. TAE with NBCA-Lip was performed in the same way as on the contralateral side. Adequate embolization was confirmed using angiography (Fig. 2). Lumbar arteries and branches of the external iliac artery were selected based on CT findings and embolized when extravasation was apparent.

\section{Endpoints}

The endpoints were the success rate of embolization, mortality rate and safety. The success rate was defined as the confirmation that arterial bleeding had ceased distal to the embolized area after the final procedure, as shown by DSA. The safety was evaluated by assessing complications that required therapy in the pelvic region according to the Society of Interventional Radiology classification [13].

\section{Results}

Table 1 lists the characteristics of the 12 patients $(7$ men and 5 women; mean age, 42 years; range, 17-74 years). The etiologies of pelvic fracture were traffic accidents $(n=4)$ and falls from a height $(n=8)$. Eleven patients met our criteria for coagulopathy. The mean ISS was 40. All patients had multiple severe injuries with an ISS $>24$, corresponding to potentially fatal conditions [14].

CECT was performed before TAE in 11 patients. Contrast extravasation was observed in 10 of these patients, indicating active bleeding. One patient without contrast extravasation was also determined to require treatment because no other obvious source of blood loss was found. One patient underwent TAE without CECT because the severity of her condition necessitated prompt treatment. Among the 12 patients, contrast medium extravasation was observed in the inferior gluteal artery in five (41.7\%), superior gluteal artery in three $(25.0 \%)$, inferior vesical artery in two $(16.7 \%)$, lateral sacral artery in two $(16.7 \%)$, iliolumbar artery in one $(8.3 \%)$, and pudendal artery in one patient $(8.3 \%)$.

All patients had concomitant injuries, including injuries to the lung, liver, spleen, kidney, adrenal gland, bladder, diaphragm, and vertebrae, as well as subarachnoid hemorrhage, subdural hemorrhage, and aortic injury.

Table 2 summarizes the procedures and results of each patient. The mean time required for bilateral occlusion of the IIAs was $17 \mathrm{~min}$ (range, 4-34 min), which was calculated from the time of DSA before and after the NBCA-Lip injection. The mean time from admission to entrance into the IR room was 89 min (range, 28-168 min).

NBCA-Lip was diluted with iodized oil (dilution range: 1/ 4-1/8 mL) via the right IIA, and $1.3 \mathrm{~mL}$ (range, 0.6-2.3 mL) was administered via the right IIA and $1.2 \mathrm{~mL}$ (range, 0.6$2.0 \mathrm{~mL}$ ) via the left IIA. The NBCA-Lip dilution ratio was not recorded in one case, and the injection dose was not recorded in another case.

All patients underwent technically successful embolization. The IIA bleeding ceased distal to the embolized area after the final procedure. However, three patients $(37.5 \%$; patients 4,6 , and 12) of the eight patients who survived beyond $24 \mathrm{~h}$ required repeat embolization using NBCA-Lip within $24 \mathrm{~h}$ after the initial procedure because extravasation was observed on CECT. Two of the 12 patients (16.7\%; patients 3 and 11) required repeat embolization during the procedure because of recanalization; repeat embolization suc- 

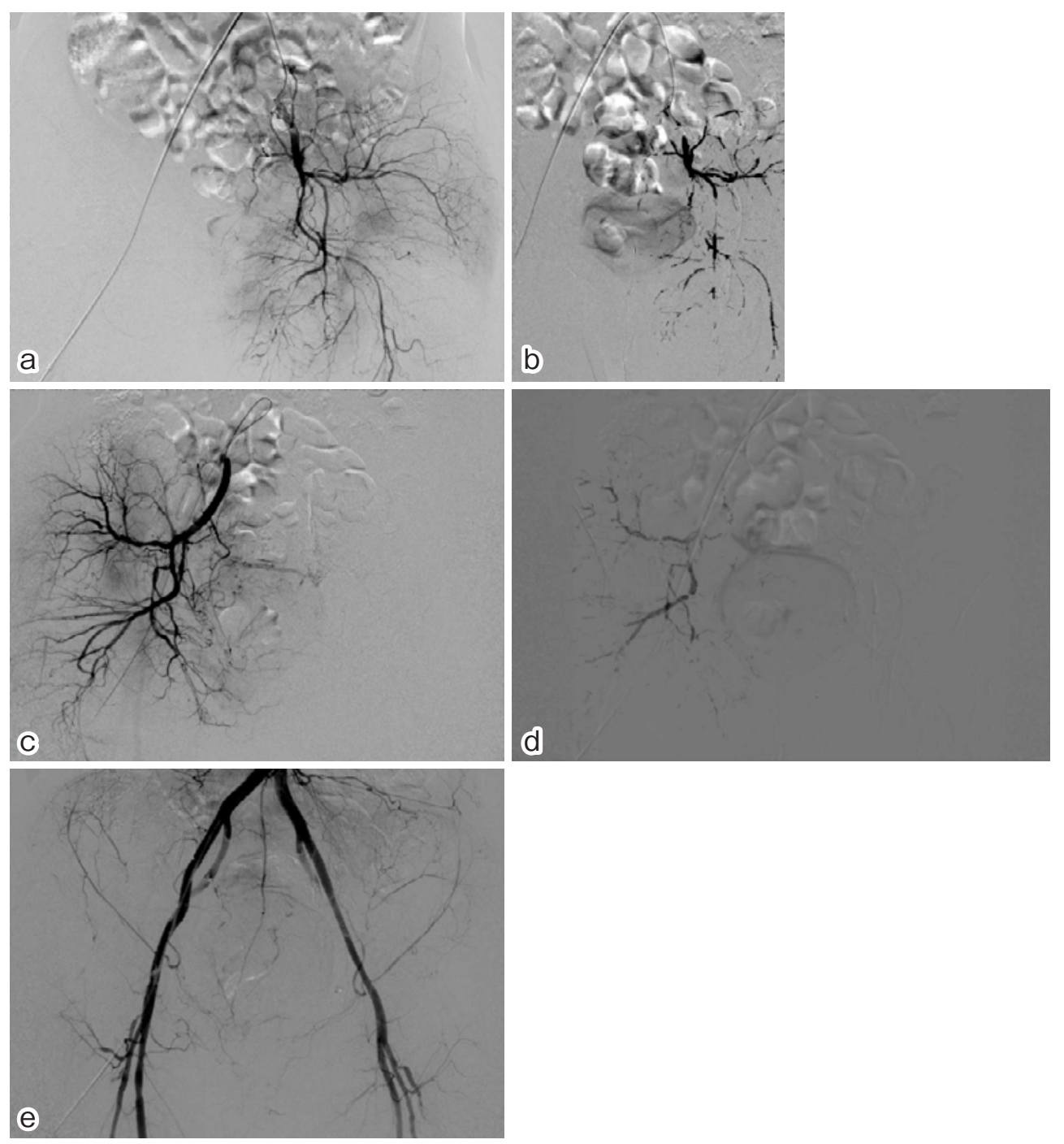

Figure 2. Imaging findings. (a) DSA of the left IIA shows vascular disruptions and calibre irregularities. (b) DSA of the left IIA shows NBCA injection from the main trunk of the left IIA. (c) DSA of the right IIA shows vascular disruptions and calibre irregularities. (d) DSA of the right IIA shows NBCA injection from the main trunk of the left IIA. (e) Adequate embolization was confirmed via pelvic angiography. DSA, digital subtraction angiography; IIA, internal iliac artery; NBCA, n-butyl2-cyanoacrylate

cessfully resolved the bleeding in these patients.

In Patient 4, despite the disappearance of arterial bleeding after the first procedure, the second angiographic examination showed recanalization of the right IIA and contrast extravasation on the left side. The vessel responsible for bleeding was the left external pudendal artery, which was then embolized with NBCA-Lip. Furthermore, the right IIA was re-embolized with NBCA-Lip, and the left fourth lumbar artery exhibited contrast extravasation and was therefore also embolized. In Patient 6, pelvic arteriography showed complete embolization of the bilateral IIAs, but contrast extravasation was still present. DSA with selective injection from the bilateral fourth lumbar arteries showed contrast extravasation; therefore, these arteries were embolized with NBCA-Lip. In Patient 12, the right pudendal artery was the crucial bleeding artery. Although the NBCA-Lip spread to the area of the bilateral IIAs, the material did not reach the bleeding point; we considered that the injection of high doses might have contributed to this. DSA showed no contrast extravasation after the procedure; however, selective arteriography of the right pudendal artery showed contrast extravasation during the second angiographic examination. Repeat embolization was required to reduce vascular spasm in Patient 4, bleeding via a collateral branch in patients 4 and 6 , and the inability of the embolic material to reach the bleeding point in Patient 12.

Four of the 12 patients (Patients 1, 7, 8, and 11) died of multiple organ failure or severe head injury within $24 \mathrm{~h}$ of procedure completion. Thus, the mortality rate in this study was $33.3 \%$. The cause of death was traumatic subarachnoid hemorrhage in patient 1 . Asphyxia due to airway obstruction or cardiac arrest was the suspected cause of death in patient 7. Hypovolemic shock due to multiple organ injury was the cause of death in patient 8 . Lung laceration with liver and 
Table 1. Demographic data, clinical status, and findings before the procedure

\begin{tabular}{|c|c|c|c|c|c|c|c|c|}
\hline Patient No. & Age (years) & Injury & coagulopathy & AIS & ISS & Contrast extravasation & $\begin{array}{c}\text { Arteries } \\
\text { of contrast extravasation }\end{array}$ & Organs of concomitant injury \\
\hline 1 & 49 & Fall & Yes & 4 & 29 & $\mathrm{~N} / \mathrm{A}$ & - & Traumatic subarachnoid haemorrhage, spleen, liver, bladder, lumbar fracture \\
\hline 2 & 18 & Fall & Yes & 4 & 36 & Yes & $\begin{array}{l}\text { Rt. lateral sacral artery } \\
\text { Rt. inferior gluteal artery }\end{array}$ & Lung, spleen, aorta \\
\hline 3 & 70 & TA & Yes & 4 & 50 & Yes & Rt. obturator artery & Liver, adrenal gland, bladder, pneumothorax \\
\hline 4 & 19 & TA & Yes & 4 & 36 & Yes & Bi. obturator arteries & Lung, pulmonary vein \\
\hline 5 & 48 & Fall & No & 4 & 33 & Yes & $\begin{array}{l}\text { Rt. lateral sacral artery } \\
\text { inferior vesical artery }\end{array}$ & bladder \\
\hline 6 & 56 & Fall & Yes & 4 & 25 & Yes & $\begin{array}{l}\text { Rt. superior gluteal artery } \\
\text { Rt. inferior gluteal artery } \\
\text { Rt. iliolumbar artery } \\
\text { inferior vesical artery } \\
\text { Bi. obturator arteries }\end{array}$ & Lung, liver, adrenal gland \\
\hline 7 & 17 & Fall & Yes & 3 & 27 & No & - & Facial bone fracture, pneumothorax \\
\hline 8 & 53 & Fall & Yes & 5 & 66 & Yes & Bi. obturator arteries & Traumatic subarachnoid haemorrhage, lung, liver, kidney, aorta \\
\hline 9 & 52 & Fall & Yes & 4 & 29 & Yes & Bi. inferior gluteal arteries & Traumatic subarachnoid haemorrhage \\
\hline 10 & 45 & Fall & Yes & 5 & 38 & Yes & Lt. superior gluteal artery & Lumbar fracture \\
\hline 11 & 34 & TA & Yes & 5 & 54 & Yes & Rt. superior gluteal artery & Lung, liver, adrenal gland, pneumothorax \\
\hline 12 & 48 & TA & Yes & 5 & 57 & Yes & $\begin{array}{l}\text { Rt. pudendal artery } \\
\text { Lt. superior gluteal artery }\end{array}$ & Traumatic subarachnoid haemorrhage, Subdural haemorrhage, lung, aorta \\
\hline
\end{tabular}

subarachnoid haemorrhage; SDH, subdural haemorrhage; N/A, not available.

Table 2. Procedures and results

\begin{tabular}{|c|c|c|c|c|c|c|c|c|c|c|}
\hline \multirow{2}{*}{ Patient No. } & \multirow{2}{*}{$\begin{array}{l}\text { Time of bilateral } \\
\text { occlusion of IIA }\end{array}$} & \multirow{2}{*}{$\begin{array}{l}\text { Time from } \\
\text { admission to enter } \\
\text { the IR room }\end{array}$} & \multirow{2}{*}{ NBCA dilution rate } & \multicolumn{2}{|c|}{ Injection dose $(\mathrm{mL})$} & \multirow{2}{*}{ Repeat embolization } & \multirow{2}{*}{ Outcome } & \multirow{2}{*}{ Cause of death } & \multirow{2}{*}{$\begin{array}{c}\text { Follow-up period } \\
\text { (month) }\end{array}$} & \multirow{2}{*}{ Complications } \\
\hline & & & & Right & Left & & & & & \\
\hline 1 & 8 & 73 & $1 / 8$ & 1 & 1 & $\mathrm{~N} / \mathrm{A}$ & Died & Traumatic subarachnoid haemorrhage & $\mathrm{N} / \mathrm{A}$ & $\mathrm{N} / \mathrm{A}$ \\
\hline 2 & 19 & 122 & $1 / 8$ & 0.6 & 0.6 & No & Survived & & 11 & No \\
\hline 3 & 20 & 86 & $1 / 7$ & 2.3 & 1.4 & No & Survived & & 4 & No \\
\hline 4 & 26 & 86 & $1 / 5$ & 1.5 & 1.5 & Yes & Survived & & 3 & No \\
\hline 5 & 8 & 89 & $1 / 8$ & 2 & 2 & No & Survived & & 38 & No \\
\hline 6 & 4 & 71 & $1 / 8$ & 0.8 & 0.8 & Yes & Survived & & 5 & Gluteal necrosis \\
\hline 7 & 34 & 75 & $1 / 8$ & $\mathrm{~N} / \mathrm{A}$ & $\mathrm{N} / \mathrm{A}$ & $\mathrm{N} / \mathrm{A}$ & Died & Asphyxia & $\mathrm{N} / \mathrm{A}$ & $\mathrm{N} / \mathrm{A}$ \\
\hline 8 & $\mathrm{~N} / \mathrm{A}$ & $\mathrm{N} / \mathrm{A}$ & $1 / 5$ & 1.5 & 1.5 & $\mathrm{~N} / \mathrm{A}$ & Died & Hypovolemic shock & $\mathrm{N} / \mathrm{A}$ & $\mathrm{N} / \mathrm{A}$ \\
\hline 9 & 30 & 79 & $1 / 8$ & 1 & 1 & No & Survived & & 36 & No \\
\hline 10 & 18 & 168 & $1 / 8$ & 1.1 & 0.7 & No & Survived & & 84 & No \\
\hline 11 & 9 & 28 & $\mathrm{~N} / \mathrm{A}$ & 2 & 2 & $\mathrm{~N} / \mathrm{A}$ & Died & Hypovolemic shock & $\mathrm{N} / \mathrm{A}$ & $\mathrm{N} / \mathrm{A}$ \\
\hline 12 & 9 & 107 & $1 / 4$ & 0.5 & 0.8 & Yes & Survived & & 4 & No \\
\hline
\end{tabular}

IR, Interventional radiology; NBCA, n-butyl-2-cyanoacrylate; N/A, not available.

adrenal gland injury resulted in hypovolemic shock in patient 11. The mean follow-up period was 23 months (range, 3-84 months). A complication related to TAE (gluteal muscle necrosis) was suspected in one of the eight surviving patients $(12.5 \%)$. Gluteal muscle necrosis is a severe adverse event in the Society of Interventional Radiology classification and requires additional treatment under general anesthesia [13]. Immediately thereafter, the patient underwent debridement of the necrotic tissue, and reconstruction with a musculocutaneous flap was performed 4 months later.

\section{Discussion}

This study assessed the efficacy and safety of nonselective bilateral embolization of IIAs with NBCA. All patients underwent technically successful embolization; however, the mortality rate was $33.3 \%$, reflecting the severity of the injuries. Only one patient developed a complication. These results are important because nonselective bilateral emboliza- tion of IIAs with NBCA could allow rapid hemostasis in severely injured patients, and complications may be tolerable.

DSA showed that arterial bleeding had ceased after the final TAE procedure in all patients; thus, the technical success rate was $100 \%$ according to our definition. This result is consistent with those of the previous studies, which reported technical success rates of $96 \%$ to $100 \%[2,15]$. Moreover, the mean time required for bilateral occlusion of the IIAs was $17 \mathrm{~min}$ (range, 4-34 $\mathrm{min}$ ). In a previous report, the mean time from the beginning of needle puncture to catheter removal was $63.3 \mathrm{~min}$, and the shortest time was $22 \mathrm{~min}$ when performing bilateral IIA occlusion with NBCA-Lip [16]. Although the definition of this time period differs from our definition, we infer that our procedure could have been performed very quickly.

The mortality rate in this study was $33.3 \%$; four patients who underwent TAE died within $24 \mathrm{~h}$ after the procedure. All four patients who died had life-threatening injuries, in addition to retroperitoneal hemorrhage from pelvic fracture. 
The remaining eight patients survived to discharge. The patients in this study had sustained multiple injuries combined with severe pelvic fracture and had a high mean ISS of 40 with a high rate of coagulopathy $(11 / 12,91.7 \%)$. In a previous study of severely injured patients that matched our study (ISS of 38.6, 18/19 patients with coagulopathy), the total survival rate was $72.1 \%$ [17]. The mortality rate in our study was not inferior to that reported in this study. For these severely injured patients, TAE with NBCA may have contributed to rapid hemostasis and recovery from shock, and may have been responsible for the low mortality rate. The median time from admission to arrival in the IR room was $86 \mathrm{~min}$ in the present study. Time from admission to TAE was an important prognostic predictor. A previous study showed that the mortality rate of patients who arrived in the IR room within $3 \mathrm{~h}$ was 14\%; however, this rate increased to $75 \%$ after $3 \mathrm{~h}$ [2]. In another study, the mortality rate of patients who arrived late in the IR room was higher than that of those who arrived within $60 \mathrm{~min}$ [18]. The survival rate could be improved if the time to arrival in the IR room could be shortened.

Repeat embolization was required within $24 \mathrm{~h}$ after the initial treatment in three patients $(37.5 \%)$. This rate of repeat embolization is consistent with those of previous reports, which ranged from $11.3 \%$ to $40.0 \%$ [19-22]. The causes of the requirement for repeat embolization were presumed to be reduction in vascular spasms, bleeding via a collateral branch, and failure of the embolic material to reach the bleeding point. The dilution rate of NBCA-Lip plays a major role in this problem. At lower concentrations, thrombosis might be inadequate, or the clot might dissolve prematurely. However, at higher concentrations, proximal embolization may occur. In this study, the dilution rate of NBCA-Lip was $1 / 4$ to $1 / 8$, which was intended to avoid proximal embolization and allow the NBCA-Lip to flow extensively. In some patients, the dilution of NBCA-Lip may have been too low or high. Therefore, it is important to embolize the cells at the optimal dilution.

One patient in the present study developed gluteal muscle necrosis after treatment (incidence of $12.5 \%$ ). Although the number of patients was small, this rate was higher than the rate of $3 \%$ to $6 \%$ reported in previous studies [23]. All patients in this study had sustained traumatic contusions to some degree, and the initial traumatic contusion might have contributed to the development of necrosis [24]. However, nonselective bilateral embolization and NBCA-Lip may be associated with an increased risk of complications. Previous reports have suggested that bilateral embolization might increase the complication rate [25] and that the powerful ischemic effect of NBCA might cause tissue ischemia [26]. Therefore, the indications for this procedure should be carefully considered for each patient.

This study has several limitations. First, this was a retrospective case series conducted at a single institution. In particular, no comparison with other embolic materials has been performed. Second, the sample size was small. Further- more, the study population was reduced because of the death of four patients within $24 \mathrm{~h}$. In future, larger prospective studies comparing NBCA and other embolic materials are required.

In conclusion, nonselective bilateral embolization of IIAs with NBCA could be a treatment of choice for hemodynamically unstable patients with severe pelvic fracture hemorrhage.

Conflict of interest: This paper was not presented at any meetings. None of the authors of this paper received financial support.

Acknowledgement: We thank Angela Morben, DVM, ELS, from Edanz Group (https://en-author-services.edanz.com/ac) for editing the draft of this manuscript.

\section{References}

1. Wubben RC. Mortality rate of pelvic fracture patients. Wis Med J 1996; 95: 702-704.

2. Agolini SF, Shah K, Jaffe J, Newcomb J, Rhodes M, Reed JF, 3rd. Arterial embolization is a rapid and effective technique for controlling pelvic fracture hemorrhage. J Trauma 1997; 43: 395-399.

3. Margolies MN, Ring EJ, Waltman AC, Kerr WS, Jr., Baum S. Arteriography in the management of hemorrhage from pelvic fractures. N Engl J Med 1972; 287: 317-321.

4. Totterman A, Dormagen JB, Madsen JE, Klow NE, Skaga NO, Roise O. A protocol for angiographic embolization in exsanguinating pelvic trauma: a report on 31 patients. Acta Orthop 2006; 77 : 462-468.

5. Velmahos GC, Chahwan S, Hanks SE, Murray JA, Berne TV, Asensio J, et al. Angiographic embolization of bilateral internal iliac arteries to control life-threatening hemorrhage after blunt trauma to the pelvis. Am Surg 2000; 66: 858-862.

6. Mizobata Y. Damage control resuscitation: A practical approach for severely hemorrhagic patients and its effects on trauma surgery. Journal of Intensive Care 2017; 5: 4.

7. Velmahos GC, Toutouzas KG, Vassiliu P, Sarkisyan G, Chan LS, Hanks SH, et al. A prospective study on the safety and efficacy of angiographic embolization for pelvic and visceral injuries. $\mathrm{J}$ Trauma 2002; 53: 303-308; discussion 308.

8. Yonemitsu T, Kawai N, Sato M, Tanihata H, Takasaka I, Nakai M, et al. Evaluation of transcatheter arterial embolization with gelatin sponge particles, microcoils, and n-butyl cyanoacrylate for acute arterial bleeding in a coagulopathic condition. J Vasc Interv Radiol 2009; 20: 1176-1187.

9. Frodsham A, Berkmen T, Ananian C, Fung A. Initial experience using N-butyl cyanoacrylate for embolization of lower gastrointestinal hemorrhage. J Vasc Interv Radiol 2009; 20: 1312-1319.

10. Valji K. Cyanoacrylates for embolization in gastrointestinal bleeding: how super is glue? J Vasc Interv Radiol 2014; 25: 20-21.

11. Hur S, Jae HJ, Lee M, Kim HC, Chung JW. Safety and efficacy of transcatheter arterial embolization for lower gastrointestinal bleeding: a single-center experience with 112 patients. J Vasc Interv Radiol 2014; 25: 10-19.

12. Spahn DR, Bouillon B, Cerny V, Duranteau J, Filipescu D, Hunt $\mathrm{BJ}$, et al. The European guideline on management of major bleeding and coagulopathy following trauma: fifth edition. Crit Care 2019; 23: 98 .

13. Khalilzadeh O, Baerlocher MO, Shyn PB, Connolly BL, Devane AM, Morris CS, et al. Proposal of a new adverse event classification by the society of interventional radiology standards of prac- 
tice committee. J Vasc Interv Radiol 2017; 28: 1432-1437.e1433.

14. Liu JS, Chiu WT, Lin LS, Hang CC. [Trauma score and injury severity score in evaluation of the trauma patients: a preliminary report of 1000 cases]. Chung Hua I Hsueh Tsa Chih 1991; 48: 297304.

15. Fangio P, Asehnoune K, Edouard A, Smail N, Benhamou D. Early embolization and vasopressor administration for management of life-threatening hemorrhage from pelvic fracture. J Trauma 2005; 58: 978-984; discussion 984.

16. Seif, Hakeem HMA, Emam, Yasser. Efficacy and procedural time of angioembolization in ongoing pelvic fracture hemorrhage. Egypt J Radiol Nucl Med 2011; 42: 185-191.

17. Huang GB, Hu P, Gao JM, Lin X. Analysis of early treatment of multiple injuries combined with severe pelvic fracture. Chin J Traumatol 2019; 22: 129-133.

18. Shinsuke T, Shigenobu M, Hideyuki M, Makoto S, Hideya N, Hiroshi I. Time to pelvic embolization for hemodynamically unstable pelvic fractures may affect the survival for delays up to $60 \mathrm{~min}$. Injury 2014; 45: 738-741.

19. Fang JF, Shih LY, Wong YC, Lin BC, Hsu YP. Repeat transcatheter arterial embolization for the management of pelvic arterial hemorrhage. J Trauma 2009; 66: 429-435.

20. Gourlay D, Hoffer E, Routt M, Bulger E. Pelvic angiography for recurrent traumatic pelvic arterial hemorrhage. J Trauma 2005; 59: 1168-1173; discussion 1173-1164.

21. Hamill J, Holden A, Paice R, Civil I. Pelvic fracture pattern pre- dicts pelvic arterial haemorrhage. Aust N Z J Surg 2000; 70: 338343.

22. Velmahos GC, Chahwan S, Falabella A, Hanks SE, Demetriades D. Angiographic embolization for intraperitoneal and retroperitoneal injuries. World J Surg 2000; 24: 539-545.

23. Wijffels DJ, Verbeek DO, Ponsen KJ, Carel Goslings J, van Delden OM. Imaging and endovascular treatment of bleeding pelvic fractures: review article. Cardiovasc Intervent Radiol 2019; 42: 10-18.

24. Suzuki T, Shindo M, Kataoka Y, Kobayashi I, Nishimaki H, Yamamoto S, et al. Clinical characteristics of pelvic fracture patients with gluteal necrosis resulting from transcatheter arterial embolization. Arch Orthop Trauma Surg 2005; 125: 448-452.

25. Travis T, Monsky WL, London J, Danielson M, Brock J, Wegelin $\mathrm{J}$, et al. Evaluation of short-term and long-term complications after emergent internal iliac artery embolization in patients with pelvic trauma. J Vasc Interv Radiol 2008; 19: 840-847.

26. Ikoma A, Kawai N, Sato M, Sonomura T, Minamiguchi H, Nakai $\mathrm{M}$, et al. Ischemic effects of transcatheter arterial embolization with N-butyl cyanoacrylate-lipiodol on the colon in a Swine model. Cardiovasc Intervent Radiol 2010; 33: 1009-1015.

Interventional Radiology is an Open Access journal distributed under the Creative Commons Attribution-NonCommercial 4.0 International License. To view the details of this license, please visit (https://creativecommons.org/licenses/by$\mathrm{nc} / 4.0 /)$. 